Cahiers $d u$ MONDE RUSSE

\section{Cahiers du monde russe}

Russie - Empire russe - Union soviétique et États indépendants

$57 / 4 \mid 2016$

Varia

\title{
Sheila Fitzpatrick, On Stalin's Team, The Years of Living Dangerously in Soviet Politics
}

\author{
Gábor T. Rittersporn
}

\section{OpenEdition}

\section{Journals}

Édition électronique

URL : http://journals.openedition.org/monderusse/10025

DOI : $10.4000 /$ monderusse. 10025

ISSN : $1777-5388$

Éditeur

Éditions de l'EHESS

\section{Édition imprimée}

Date de publication : 1 octobre 2016

Pagination : 948-952

ISBN : 978-2-7132-2542-0

ISSN : $1252-6576$

\section{Référence électronique}

Gábor T. Rittersporn, «Sheila Fitzpatrick, On Stalin's Team, The Years of Living Dangerously in Soviet Politics », Cahiers du monde russe [En ligne], 57/4 | 2016, mis en ligne le 01 octobre 2016, Consulté le 24 septembre 2020. URL : http://journals.openedition.org/monderusse/10025 ; DOI : https://doi.org/ 10.4000/monderusse. 10025 
loyalty" (p. 114); "False Prophets and Closet Rebels" (p. 159). Even the book's title becomes a mystery, because what separates anguish from anger in the historical record is rarely made apparent. This is an author that likes to tease the reader more than to help.

A strong message of the book is that the attempt to create a planned economy led to chaos, and that the Bolsheviks' totalitarian aspirations were continually subverted by the counter-strategies of the subjects. It's a fair point, although not new. It also leaves something out: the planned economy that the Bolsheviks created was, for a considerable time, a success. Not a success, perhaps, for the ordinary workers and peasants who had to make it work, but still a success for its creators. Lenin and Stalin wanted a centralized command system for the mass production of things, especially of the things that they saw as means of power in the world: locomotives, lathes, power stations, ships, tanks, and planes. Here was the system, even if many people experienced it as chaos, and the system worked, turning a third-class economy into a first-class military power. After many years the system collapsed, but not before it had qualified as one of the most durable of modern dictatorships.

This important book is certain to have an influence on future scholarly research and on the teaching of Russia's social history. How widespread that influence will be is not entirely clear. For some readers it will be enough to dip into the pool and catch hold of some fascinating gem. Others will require plenty of deep breathing followed by prolonged immersion to grasp Rittersporn's project in its entirety.

\section{Mark Harrison \\ University of Warwick}

\section{Sheila FITZPATRICK \\ On Stalin's Team \\ The Years of Living Dangerously in Soviet Politics}

Princeton et Oxford : Princeton University Press, 2015, 364 p.

Il a fallu attendre longtemps avant que les historiens de l'époque soviétique ne commencent à accepter l'idée que Stalin n'agissait pas dans un vacuum. Nombre d'adeptes de la confrérie ne sont pas encore tout à fait certains que le dictateur ait eu à trouver un modus vivendi avec la réticence des paysans à se plier à sa politique rurale, ni s'il avait été bien avisé d'adapter sa politique pénale à la résilience d'une criminalité sociale engendrée par le régime lui-même ou s'il avait dû composer avec un appareil de l'État-parti, où les réseaux d'assistance mutuelle des cadres se reconstituaient et persistaient, même après des purges meurtrières. De ce fait, l'approbation unanime par les collègues d'un ouvrage qui montre que l'équipe dirigeante du père des peuples soviétiques n'était pas qu'un instrument docile n'est pas acquise.

Pourtant, Sheila Fitzpatrick argumente bien son cas. Elle identifie un noyau dur au sein du Politbureau du Parti qui, ayant survécu à toutes les vicissitudes d'une 
vingtaine d'années d'histoire soviétique, réussit, après la guerre, à peser de plus en plus sur les orientations du régime. Le groupe émergea dans les années 1920, ses premiers membres étaient Viačeslav Molotov, Kliment Vorošilov, Lazar' Kaganovič, Anastas Mikojan, Andrej Andreev, ainsi que, jusqu'à leur mort respectivement en 1934, 1935, 1937 et 1946, Sergej Kirov, Valerjan Kujbyšev, Grigorij Ordžonikidze et Mihail Kalinin. Nikita Hruščev, Georgij Malenkov, Lavrentij Berija et Andrei Ždanov (mort en 1948) furent cooptés dans les années 1930. Le statut des membres varia avec le temps. Ainsi, Kalinin, Vorošilov et Kaganovič ne furent pas toujours dans les bonnes grâces et les maladies d'Andreev l'éloignèrent du groupe. Molotov et Mikojan furent écartés dans les mois qui précédèrent la mort de Stalin, et Fitzpatrick pense (avec d'autres auteurs, et Hruščev aussi) qu'à ce moment-là, leur vie était en danger.

Malgré tout, il ne semble pas que l'équipe ait vécu aussi dangereusement que le sous-titre le suggère. Les membres du noyau dur ont mis la main à la pâte pendant l'épuration de l'appareil entre 1936 et 1938. Et si le cercle de leurs amis, de leurs collaborateurs et de leurs parents ne fut pas épargné, la plupart du temps les dignitaires se gardèrent bien de plaider la cause de leurs proches, ce qui leur évita d'être soupçonnés de pactiser avec les prétendus ennemis du peuple. Mikojan a la réputation de quelqu'un qui a tenté de sauver des camarades. Or, cette réputation, c'est lui-même qui se l'est construite dans ses Mémoires rédigés une trentaine d'années plus tard, à une époque qualifiée de « végétarienne » par Nadežda Mandel'šstam.

Dans les meilleurs chapitres du livre, Fitzpatrick décrit la constitution et le fonctionnement de l'équipe dans les années 1920 et 1930. Les comparses s'étaient connus au gré de leurs activités révolutionnaires, des fortunes de la Guerre civile et des missions dans les structures naissantes de l'appareil. Ils jouèrent un rôle important dans les luttes contre l'opposition de gauche aux côtés de Stalin et firent leurs preuves comme administrateurs énergiques. C'est en gérant les affaires de la collectivisation désastreuse de l'agriculture et de la construction quelque peu aventureuse d'une nouvelle industrie qu'ils élaborèrent leurs pratiques de concertation de leurs actions, qui allaient de l'emploi non déguisé de la violence à l'organisation du travail d'une multitude d'institutions. Les intrigues contre les rivaux réels et imaginaires du groupe faisaient partie prenante de ces pratiques.

La coterie s'était soudée en manœuvrant contre une dissidence, somme toute timide, au sein du Politbureau, du gouvernement et du Comité central et en se battant contre ceux que ses membres prenaient pour les adversaires de leur politique économique hasardeuse. Si Fitzpatrick évoque le document dans lequel Martemjan Rjutin exposait en 1932 ce que certains militants de base comprirent comme un tournant bonapartiste, elle ne s'arrête pas sur le désarroi dans les rangs inférieurs du Parti, qui a certainement contribué à la chasse aux trotskistes fantomatiques et autres oppositionnels chimériques parmi les bolcheviks. Cependant, l'imagerie partagée d'un danger contre-révolutionnaire et ses conséquences pour le régime furent inséparables de la formation de l'équipe et de son auto-perception d'être la seule légataire authentique de ce qu'elle avait pris comme étant l'héritage d'Octobre. 
Les membres individuels étaient responsables des plus importantes administrations centrales et régionales. Ils représentaient les intérêts de leurs institutions et ils faisaient de leur mieux pour les faire valoir au sein de la direction. Ordžonikidze était particulièrement insistant en défendant l'industrie lourde et ses alliés au sein de l'appareil, si bien qu'il provoquait systématiquement des conflits, surtout avec Stalin. Apparemment, ses collègues jouaient plus subtilement. Malheureusement, Fitzpatrick n'éclaire pas le lecteur sur ce point. Elle n'essaie pas de voir si ses héros avaient recruté des clientèles dans leurs administrations, pas plus qu'elle ne tente d'expliquer pourquoi ils ne l'ont pas fait, s'il était avéré que les plus hauts dignitaires n'avaient pas construit de réseaux de fidèles autour de leurs personnes. Il se peut qu'ils aient dû éviter qu'on ne les soupçonne d'organiser des fractions. Il se peut également qu'ils n'aient pas eu besoin de réunir de coteries, car c'est l'équipe même qui devait fonctionner comme un réseau de solidarité face aux cliques qui se formaient dans les administrations régionales et centrales. Arch Getty a montré l'importance de ces dernières, mais ces clans et leurs relations avec le groupe dirigeant de Stalin ne sont pas traités dans le livre.

L'immense autorité de Stalin apparaît dans l'ouvrage comme allant de soi. Elle a sans doute commencé à aller de soi pour ses fidèles au cours de la crise qui a fait suite à la collectivisation et à l'industrialisation et elle n'a fait que s'amplifier pour prendre d'énormes proportions plus tard. Cependant, il a bien fallu l'acquérir et on peut se demander si, parfois, il n'a pas fallu la reconquérir. Les sources montrent que la crise du début des années 1930, les manœuvres diplomatiques à la fin de la décennie, la politique agraire, les mesures pour discipliner la main-d'œuvre, la criminalisation de modes de comportements populaires, le conflit désastreux avec la Finlande, l'occupation de nouveaux territoires et les interventions du Leader suprême aux décisions du haut commandement pendant la guerre n'ont pas suscité l'enthousiasme unanime des cadres, des spécialistes de l'économie, des diplomates, des militaires et de la population. Les lettres que Stalin et Kaganovič ont échangées en juillet 1932 révèlent que la loi qui devait punir par la mort les atteintes aux biens publics a divisé le noyau dur. Fut-ce le seul moment d'hésitation de l'équipe?

Le rôle de rassembleur de Stalin pendant les conflits avec l'opposition dans les années 1920 ainsi que son talent à organiser l'appareil et à le faire fonctionner dans des situations critiques jetèrent les fondements de son autorité. Il était celui qui savait comment sortir des crises provoquées par ses politiques. De même, il était celui qui semblait en mesure de faire de l'ordre dans le travail chaotique d'institutions et d'administrations dont la marche n'était pas soumise à des règles fermement établies, celui encore qui avait des recettes pour discipliner le personnel qui ne se soumettait pas aux normes non écrites de l'État-parti. Stalin parvenait à dicter à ses acolytes sa propre lecture de certaines situations, de crises et de leurs origines, de l'ordre qui devait émerger du chaos ainsi que des raisons de la conduite des cadres et des répercussions hypothétiques. Cependant, il serait intéressant d'avoir une idée de ce que ses collègues croyaient comprendre avant qu'il n'impose sa vision. 
Fitzpatrick tente de reconstruire l'itinéraire de l'équipe après la guerre et c'est un grand mérite de l'ouvrage. Elle réfléchit à la question de savoir pourquoi les compères ont réussi à prendre en main les affaires du pays sans le moindre problème déjà quand Stalin était mourant, à continuer à les gérer comme de si rien n'était après la mort du Dirigeant suprême qu'on s'imaginait irremplaçable, à entamer des changements politiques sans hésiter et à arriver à un consensus remarquable, même quand il s'est agi de s'élever contre un camarade de longue date comme Berija. Pour Fitzpatrick, l'équipe avait l'habitude de travailler sans Stalin depuis les années 1920 et elle avait pris à sa charge la gestion de toujours plus de questions après la guerre. De surcroît, elle était de plus en plus en capacité d'ignorer les mouvements d'humeur du chef. Elle fit ainsi preuve d'une remarquable solidarité quand il voulut exclure Molotov et Mikojan du groupe. Dès lors, il n'est pas certain que les comparses auraient consenti à la purge que Stalin semblait préparer à la fin de 1952, tout comme il n'est rien moins sûr que la politique mise en œuvre par la clique ait toujours correspondu aux vœux du chef. D'où, précisément, ses manœuvres pour lancer une purge peu avant sa mort.

La qualité des chapitres sur la formation du groupe et sur son travail jusqu'en 1936 est en partie redevable à celle des sources disponibles sur cette période. La correspondance de Stalin avec ses lieutenants pendant les périodes plus ou moins longues qu'il passait en vacances montre à quel point il faisait confiance à ses fidèles. Elle révèle également les questions qui l'intéressaient le plus, ainsi que ses désaccords avec tel ou tel autre collaborateur. Les lettres aident Fitzpatrick à lire d'autres documents. Elle rencontre davantage de difficultés quand elle travaille sur la fin des années 1930, sur la guerre et surtout sur l'après-guerre, car elle doit prêter une attention toujours plus grande aux Mémoires des acteurs et de leurs proches ainsi qu'à des entretiens que certains protagonistes accordèrent à des confidents dans les années 1970 et plus tard.

Fitzpatrick est consciente des problèmes que posent les écrits intimes, en particulier quand ils ont été préparés à la publication par les proches des auteurs, ou quand ce sont les enfants des dignitaires qui racontent ce qu'ils savaient dans les années 1980 et 1990 des agissements de leurs pères dans un passé lointain et du milieu dans lequel ils exerçaient. Malgré tout, elle succombe parfois à la tentation de citer verbatim des conversations auxquelles les mémorialistes ne pouvaient pas avoir assisté et que, parfois, ils ne tenaient même pas de seconde main. Il n'est pas simple de distinguer les faits indiscutables d'une tradition orale qui a émergé vers la fin des années 1950 .

Aussi, on peut s'interroger quant à la véracité de certaines confidences que Fitzpatrick reprend d'un Molotov ou d'un Kaganovič, dont les ellipses et les omissions évidentes sur nombre de questions incitent à la plus grande prudence. De plus, leur interlocuteur, Feliks Čuev, n'a pas enregistré ces longues conversations. Il les a notées de mémoire en tentant de reproduire mot pour mot ce qu'il avait entendu. Rien n'est moins sûr qu'il ait réussi à restituer les monologues prolixes de ses protagonistes, qu'il a ensuite coupés et recollés selon les sujets évoqués, comme il l'indique dans ses recueils, en publiant parfois le montage d'entretiens 
ayant eu lieu à trois ou quatre occasions différentes entre le début des années 1970 et les années 1980. Il avait de quoi puiser (et de quoi omettre) dans ses nombreuses conversations avec Molotov, cent quarante à l'en croire.

Il est une question que les mémorialistes illustres et leurs proches ne mentionnent guère et que Fitzpatrick ne fait qu'effleurer au détour d'une phrase, c'est celle de la grande vague de terreur qui s'est traduite par l'exécution de centaines de milliers de personnes et la déportation de centaines de milliers d'autres entre août 1937 et novembre 1938. Il s'agissait de simples citoyens soviétiques massacrés en masse, à la différence des nombreuses victimes plus ou moins haut placées, dont le sort préoccupa les anciens dignitaires, leurs amis et leurs parents. Hruščev avait de bonnes raisons de ne souffler mot sur son rôle à Moscou et en Ukraine et il n'est pas étonnant que le fils de Berija ne se soit pas étendu sur la responsabilité de son père dans la terreur en Géorgie et, plus tard, à l'échelle du pays. Il aurait sans doute été possible de retrouver les traces des exploits du groupe dans les recueils de documents et dans les ouvrages sur la Terreur et de les mettre dans la perspective du livre.

La description de l'équipe en termes de réseau social constitue un moment fort intéressant de l'ouvrage. Jusqu'à la seconde moitié des années 1930, Stalin et ses acolytes se fréquentaient, accompagnés de leurs familles et de quelques amis qui n'appartenaient pas au cercle. Ils fêtaient ensemble l'anniversaire de tel ou tel ou celui de la révolution, ils se rassemblaient dans les datchas des uns et des autres, ils passaient des vacances ensemble. Leurs enfants grandissaient ensemble, avec la progéniture d'autres dignitaires, et il n'était pas rare qu'ils se marient entre eux. Cette intimité s'estompa vers la fin des années 1930. Fitzpatrick attribue l'affaiblissement des liens à la purge dans les milieux de l'élite entre 1936 et 1938 et au climat de suspicion qui en résulta et affecta les survivants.

Chose assez rare dans le métier, la qualité littéraire de l'ouvrage procure un grand plaisir. Le style est vif, le récit parfois drôle et empli de formules originales. Fitzpatrick compte sur une audience plus large que celle des consommateurs universitaires de travaux spécialisés. Espérons que ses vœux soient exaucés. Toutefois, il est à craindre que la lecture de son livre suppose plus de connaissances de l'histoire soviétique et de ses personnages que celles du grand public. Quoi qu'il en soit, il est sûr que la confrérie des historiens en apprendra beaucoup.

Gábor T. Rittersporn

CNRS

\section{Denis SKOPIN}

La photographie de groupe et la politique de la disparition dans la Russie de Staline Paris : L’Harmattan (Coll. : Esthétiques), 2015, 236 p.

Denis Skopin, a philosopher in St. Petersburg, Russia, earned his doctorate in aesthetics at l'Université Paris VIII. Theoretically informed by the French philosopher Gilbert Simondin (1924-1989), Skopin offers a meditation in La 